\title{
Evaluación de la primera implementación del plan de rotación de puestos de aprendizaje en la universidad tecnológica de Puerto Peñasco desde la perspectiva del formador-empresario
}

\author{
Ramsés Everardo Moreno-Murrieta \\ ramsesmoreno@utpp.edu.mx \\ María Julisa Mendivil-Yescas \\ julisamendivil@utpp.edu.mx \\ Lady Priscila Solorzano Barba \\ ladysolorzano@utpp.edu.mx
}

Universidad Tecnológica de Puerto Peñasco

Puerto Peñasco - México

\section{RESUMEN}

La Universidad Tecnológica de Puerto Peñasco realizó la primera implementación del Plan de Rotación de Puestos de Aprendizaje del Modelo de Formación Dual durante el período de alternancia escuela-empresa en el año 2019. Con el objetivo de evaluar esta implementación desde la perspectiva del formador-empresario, se analiza la vinculación entre empresa y universidad, el desempeño de los aprendices y de los beneficios adquiridos y otorgados por la empresa, para la determinación de la calidad de su participación en la etapa dual. Los formadores-empresarios consideran que la participación en la primera implementación fue satisfactoria, los aprendices reforzaron los conocimientos adquiridos en la universidad, y la empresa contribuyó al desarrollo de las competencias laborales de los estudiantes.

Palabras clave: modelo de formación dual; plan de rotación de puestos de aprendizaje; alternancia escuela-empresa. 


\title{
Evaluation of the first rotation planning and implementation of learning positions of the Technological University of Puerto Peñasco from the businessmen-trainning perspective
}

\begin{abstract}
The Technological University of Puerto Peñasco has done the first rotation planning and implementation of learning positions on the Dual training model during the period of alternation school-company in 2019. The objective of this research was to evaluate this implementation from the perspective of the businessmen- training. They analyzed the relation between the company and the university, the performance of the trainers, and the acquired benefits and granted by the company. They also took into consideration the determination of the quality in their achievement in the duals stage. The businessmentraining considered that the participation in the first performance was satisfactory. The trainers reinforced their acquired knowledge in the university, and the company contributed to the development of the labor skills of the students.
\end{abstract}

Keywords: dual training model; rotation plan of learning positions; school-company alternation.

Artículo recibido: 05 octubre. 2021 Aceptado para publicación: 02 noviembre 2021 Correspondencia: ramsesmoreno@utpp.edu.mx Conflictos de Interés: Ninguna que declarar 


\section{INTRODUCCIÓN}

La implementación formal del Modelo Mexicano de Formación Dual y en Alternancia (MMFD) en educación superior es el resultado del análisis de las prácticas de formación dual llevadas a cabo desde el año 2013 en distintas instituciones, entre ellas las pertenecientes al Tecnológico Nacional de México (Romero, 2016), a la Coordinación General de Universidades Tecnológicas y Politécnicas (CGUTyP) y en varias universidades públicas estatales y privadas (Armendáriz, 2018). La sistematización de estas experiencias por parte de la CGUTyP permitió integrar la metodología de implementación del MMFD y su posterior promoción en las Universidades Tecnológicas y Politécnicas del país con el fin de homologar y diseminar su práctica (FESE, 2019).

El MMFD tiene como eje operativo rector el Plan de Rotación de Puestos de Aprendizaje (PRPA) el cual diseñado por el conjunto Escuela-Empresa define y programa los distintos puestos de trabajo dentro de la empresa en los que el aprendiz-alumno desarrolla o adquiere las competencias laborales, esto con el apoyo de un formador-empresario para cumplir con el objetivo de la formación dual (Covarrubias, 2018; Cuenca, 2018; GIZ, 2019). Si bien la experiencia de la implementación de un modelo de formación dual en la educación superior ha sido dada a conocer por algunas instituciones de educación superior en México (Castellanos-Rodríguez, Mendívil-Yescas \& Moreno-Murrieta, 2020; Manzur, Martínez \& Cortés, 2019; Rocha \& Alemán, 2019; Vargas, 2016; Velarde \& Medina, 2014), únicamente Covarrubias (2018) ha evaluado la percepción de los formadores-empresarios después de su participación en un esquema formación dual.

El objetivo de este trabajo es evaluar la primera implementación del PRPA, desde la perspectiva del formador-empresario, en la Universidad Tecnológica de Puerto Peñasco (UTPP) por medio del análisis de la vinculación empresa-universidad, la evaluación de los aprendices (según los formadores), y de los beneficios adquiridos y otorgados por la empresa para la determinación de la calidad de su participación en la etapa dual.

\section{ESTRATEGIAS METODOLÓGICAS O MATERIALES Y MÉTODOS}

Partiendo de un enfoque exploratorio cualitativo se realizó un análisis transversal de los primeros dos periodos de implementación del PRPA en la UTPP durante el año 2019, para ello se aplicó una entrevista estructurada a una muestra de cuatro formadoresempresarios seleccionados de manera no aleatoria por conveniencia, de un total de siete empresas participantes en el modelo dual (cada empresa con un formador). Para el primer 
periodo, enero-abril, se seleccionaron dos empresas, una del giro de servicio de catering (con cuatro aprendices del TSU en Gastronomía) y otra de soporte técnico e informático (con tres aprendices del TSU en Tecnologías de la Información y Comunicación); para el segundo periodo, septiembre-diciembre, una empresa del giro restaurantero (con cinco aprendices del TSU en Gastronomía) y otra de servicios de instalación y mantenimiento (con 10 aprendices del TSU en Mantenimiento Industrial - Área Refrigeración).

La entrevista estructurada a los formadores-empresarios fue adaptada de Covarrubias (2018) y se orientó al análisis de tres ejes principales: la empresa y su vinculación con la universidad (con nueve preguntas), el desempeño de los aprendices (con siete preguntas), y las prácticas de contratación y capacitación al personal (con cuatro preguntas). Adicionalmente se incluyeron tres preguntas para una evaluación global de la experiencia de la participación de la empresa en el modelo de educación dual.

\section{RESULTADOS Y DISCUSIÓN}

Los resultados obtenidos se presentan en función de los tres ejes mencionados, mientras que la evaluación global de la experiencia del formador-empresario en el modelo dual se aborda en la sección de conclusiones.

\subsection{La empresa y su vinculación}

Los formadores-empresarios indicaron que en todos los casos la universidad inició con el acercamiento para la implementación del modelo dual, coincidiendo en haber participado previamente en alguna etapa de estadía profesional con la universidad y siendo esta la única participación de la empresa en cualquier otro programa de vinculación con alguna institución de educación superior. Los objetivos que los impulsaron a participar en el MMFD fueron: apoyar con conocimientos prácticos a los aprendices, contribuir con egresados mejor preparados en el perfil profesional de su carrera y, formar y capacitar a futuros trabajadores de su empresa.

Los puestos de rotación que se definieron en conjunto por la universidad y la empresa fueron: coordinador de eventos, atención a clientes y chef, esto en el caso la empresa del giro de servicio de catering; soporte técnico, gerente de proyecto y desarrollo de software, para la empresa de soporte técnico e instalación; administración y operación de cocina, servicio a clientes y gestión de administración, en la empresa del ramo restaurantero; y finalmente, la rotación en los puestos de auxiliar de técnico en refrigeración, técnico en refrigeración y proyectista, en la empresa del giro instalación y mantenimiento. 
La comunicación y coordinación que se generó entre los empresarios y la universidad para el control del aprendizaje y los resultados académicos de los aprendices fue a través de los tutores representantes de cada programa educativo, mediante reuniones entre los formadores-empresarios y docentes, comunicación vía telefónica o correo electrónico, y en especial las visitas presenciales a las empresas (una vez por semana) para evaluar el avance programado para los aprendices.

\subsection{El desempeño de los aprendices}

Los formadores-empresarios manifestaron no participar en la selección de los aprendices, la universidad a través del departamento de vinculación y la recomendación de los tutores de cada programa educativo asignaron a los alumnos para su participación en el modelo. Las características o competencias que la empresa demandó de los aspirantes a aprendices fueron las competencias del saber ser, principalmente valores como el compromiso, el trabajo en equipo y una buena actitud.

En cuanto a las competencias y aprendizaje que se desarrollaron en la empresa, en cada puesto de rotación se manifestó la adquisición de la competencia planeada, aunque no de manera uniforme (unos con mayor o menor grado de desempeño), en todos los casos la participación del aprendiz-alumno se consideró como un aporte a la productividad de la empresa.

\subsection{Prácticas de contratación y capacitación de personal}

Desde la perspectiva del formador-empresario hay diferencia entre los alumnos del MMFD y los que participaron en otro tipo de vinculación anteriormente (alumnos en etapa de estadía profesional). Los aprendices, bajo las mismas condiciones que un trabajador, manifestaron mejor desempeño en el desarrollo de las competencias y actitudes debido a un mayor nivel de compromiso en la ejecución de las actividades dentro de la empresa.

En cuanto a las prácticas de contratación, los formadores-empresarios han ofrecido puestos de trabajo a los alumnos egresados del MMFD, concretándose cuatro contrataciones de manera definitiva y cinco de manera eventual, nueve de un total de 22 aprendices.

\section{CONCLUSIÓN O CONSIDERACIONES FINALES}

Esta evaluación de la implementación del PRPA en la Universidad Tecnológica de Puerto Peñasco es un caso particular y no generalizable dadas las condiciones del tipo de estudio 
y las limitaciones de la institución para llevarla a cabo a una mayor escala, sin embargo el lograr recabar la perspectiva de los primeros formadores-empresarios permite enriquecer y fortalecer las futuras implementaciones del modelo promovido por la CGUTyP.

En términos generales los formadores-empresarios consideran que la participación en la primera implementación del MMFD en la universidad fue satisfactoria, los aprendices en la alternancia escuela-empresa reforzaron los conocimientos adquiridos en las aulas, y la empresa contribuyó al desarrollo de las competencias laborales de los estudiantes.

Los empresarios coinciden que el sistema dual es enriquecedor y gratificante en la formación académico-laboral del aprendiz debido a que éste toma el rol de empleado dentro de la empresa, se responsabiliza de las actividades y tareas asignadas, y adquiere mayores conocimientos prácticos por la rotación de puestos de aprendizaje.

En cuanto a futuras participaciones, los formadores tienen la intención de ser parte de las próximas generaciones de aprendices del MMFD por el interés de contribuir a la formación y crecimiento profesional del estudiante, destacando la importancia de contar con técnicos profesionales mejor capacitados en la región. La recomendación de los formadores-empresarios se centró en la incorporación de las competencias del saber ser dentro del proceso de enseñanza-aprendizaje del modelo dual, tanto en la escuela y en la empresa, ya que consideran que la disponibilidad para aprender, la buena actitud y el trabajo en equipo son condicionantes para la adquisición de las aptitudes en el campo laboral.

\section{LISTA DE REFERENCIAS}

Armendáriz M. (2018). Modelo Mexicano de Formación Dual. Sesión del Diplomado Metodología Curricular. Dirección General de Educación Media Superior y Superior.

UAGro. http://educacion.uagro.mx/index.php?option=com_content $\& v i e w=\operatorname{article} \& i d=7$ $\&$ Itemid $=\&$ jsmallfib $=1 \&$ dir=JSROOT//Documentos $/$ Diplomado+Metodolog\% $\mathrm{C}$ 3\%ADa+Curricular/Sesi\%C3\%B3n+1.+Metodolog\%C3\%ADa+curricular\&sort _by=changed\&sort_as=desc

Castellanos-Rodríguez, V., Mendívil-Yescas, M. J., \& Moreno-Murrieta, R. E. (2020). Evaluación de la implementación del plan de rotación de puestos de aprendizaje desde la perspectiva del aprendiz. Universo de la Tecnológica. Año XII. Volumen 
III, $\quad$ Edición $\quad 36 . \quad$ Mayo-Agosto 2020.

https://revista.utnay.edu.mx/index.php/ut/article/download/8/17

Castro, M. (2016). Plan de sucesión en cargos críticos de profesionales especialistas y su impacto en la percepción de rotación voluntaria.

Covarrubias A. (2018). El modelo de formación dual de Baja California: ¿Una nueva forma de articulación entre educación y empleo? [Tesis de Maestría, El Colegio de la Frontera Norte, A. C., Tijuana, México]. https://www.colef.mx/posgrado/tesis/20161253/

Cuenca A. (2018). Los desafíos y avances de la educación en México en el siglo XXI. Cotidiano-Revista de la Realidad Mexicana, 33(208). http://www.imced.edu.mx/Portal/files/educacion_en_mexico.pdf

Díaz Peña, K. P., Agudelo Rubiano, Y. P., \& Pardo Sánchez, W. F. (2018). Rotacion de personal, un factor a evaluar en Inmobiliaria e Inversiones Chico ltda.

FESE (2019). Marco de Referencia para la Formación Dual en la Educación Superior en México. Formación Dual en al Educación Superior. 2ed. Fundación Educación Superior-Empresa A.C. http://descargas.fese.mx/index.php?id=22\#

GIZ (2019) Manual para el Instructor/Coordinador e Instructor/Formador. Ciudad de

Haddad Morote, J. D., \& Nuñez Chasquero, J. C. (2017). Evaluación de técnicas de ventas y manejo de rotación de inventarios y su influencia en las ventas de Bodegas en Lima Metropolitana 2017.

Manzur S., Martínez M., \& Cortés A. (2019). El sistema de educación dual en México: caso Universidad Politécnica del Valle de Toluca. Dilemas Contemporáneos: Educación, $\quad$ Política $\quad$ y https://www.dilemascontemporaneoseducacionpoliticayvalores.com/index.php/d ilemas/article/view/1606

México. https://www.giz.de/en/downloads/Manual-para-el-Instructor.pdf

Otero Tavara, M., \& Torres Canchanya, K. (2016). Plan de mejora de la gestión de rotación de personal y siniestralidad para la división de operaciones de una empresa contratista minera.

Ramírez Broncano, L. P. (2018). Propuesta de un plan de capacitación para disminuir la rotación de colaboradores del área de operaciones de la Linea 1 del Metro de Lima 2018. 
Rocha, M. \& Alemán L. (2019). Programa escuela-empresa, antecedente de formación dual: caso Universidad Tecnológica del norte de Aguascalientes, México. Quipukamayoc, 27(54), pp. 91-96. https://doi:org/10.15381/quipu.v27i54.16263.

Rodríguez Ponce, A. P. (2020). Estudio de los factores que originan la rotación del personal. Caso: ECUATRAN SA (Bachelor's thesis, Pontificia Universidad Católica del Ecuador).

Ruiz Fierro, A. (2019). Evaluación de las estrategias de gestión del capital humano e impacto organizacional en la alta rotación de personal en flores ipanema finca guaymaral sas (Doctoral dissertation).

Taco, D., \& Carlos, J. (2018). Estudio del impacto de la rotación de personal sobre los índices de evaluación del desempeño de los servidores del Gobierno Autónomo Descentralizado Municipal cantón Alausí, periodo 2016-2017 (Bachelor's thesis, Escuela Superior Politécnica de Chimborazo).

Téllez, D. P. P., González, J. S., \& Rodríguez, A. F. S. (2016). Identificación de las variables que influyen en la rotación de los colaboradores del Proyecto P y P de CODESS y generación de un plan de acción para disminuir el indicador de rotación.

Vargas, M. (2016). 1. Educación dual y educación en alternancia. En M. V. Leyva, Educación Dual: Experiencias exitosas de vinculación escuela-empresa (pp. 1520). Tijuana:

ILCSA. https://www.researchgate.net/publication/313102354_Educacion_Dual_Experie niencias_exitosas_de_vinculacion_escuela-empresa

Velarde, L., \& Medina, A. (2014). Sistema Dual de Aprendizaje: Un modelo educativo dinámico para una formación dual. En L. Pérez Ríos, E. Huerta Mascote, M. Ferrer Almaraz, \& M. A. Núñez Ledesma, Educación Handbook T-IV (pp. 196207). Valle de Santiago: Ecorfan-México. https://www.ecorfan.org/handbooks/Educacion\%20T-

IV/ARTICULO\%2020.pdf 